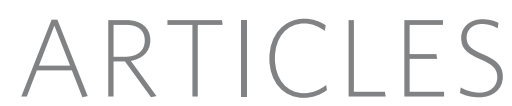

\title{
High harmonic interferometry of multi-electron dynamics in molecules
}

\author{
Olga Smirnova ${ }^{1,2}$, Yann Mairesse ${ }^{1,3}$, Serguei Patchkovskii ${ }^{1}$, Nirit Dudovich ${ }^{1,4}$, David Villeneuve ${ }^{1}$, Paul Corkum ${ }^{1}$ \\ \& Misha Yu. Ivanov ${ }^{1,5}$
}

High harmonic emission occurs when an electron, liberated from a molecule by an incident intense laser field, gains energy from the field and recombines with the parent molecular ion. The emission provides a snapshot of the structure and dynamics of the recombining system, encoded in the amplitudes, phases and polarization of the harmonic light. Here we show with $\mathrm{CO}_{2}$ molecules that high harmonic interferometry can retrieve this structural and dynamic information: by measuring the phases and amplitudes of the harmonic emission, we reveal 'fingerprints' of multiple molecular orbitals participating in the process and decode the underlying attosecond multi-electron dynamics, including the dynamics of electron rearrangement upon ionization. These findings establish high harmonic interferometry as an effective approach to resolving multi-electron dynamics with sub-Ångström spatial resolution arising from the de Broglie wavelength of the recombining electron, and attosecond temporal resolution arising from the timescale of the recombination event.

Structure-induced interference minima in photo-ionization were predicted for $\mathrm{H}_{2}{ }^{+}$by Cohen and $\mathrm{Fano}^{1}$, providing the foundation for extracting structural information from photoelectron spectra. Similar interference in photo-recombination, which is the last step in high harmonic emission, has been predicted to produce minima in the harmonic spectra of $\mathrm{H}_{2}{ }^{+}$(ref. 2), stimulating experiments on using high harmonic generation to image molecular structures ${ }^{3-11}$. These experiments benefit from the ability to induce and control molecular alignment in a gas ${ }^{12,13}$. The minima have indeed been found in the harmonic spectra of the $\mathrm{CO}_{2}$ molecule, but at vastly different positions in different experiments ${ }^{4,5,8,9}$. The structure of the molecule is the same, so why does the minimum appear at different places? We find that it shifts with the laser intensity and its position and mobility reflect attosecond multielectron dynamics in the ion, including electron rearrangement upon ionization.

It has been assumed that structures in the harmonic spectra reflect the geometry of the highest occupied molecular orbital $(\mathrm{HOMO})^{3-5,8,9,14-18}$. Indeed, strong-field ionization is exponentially sensitive to the ionization potential $I_{\mathrm{p}}$, suggesting that after ionization the molecular ion is left in its ground electronic state (electron removal from HOMO in the Hartree-Fock picture). In this case, even though rearrangement has occurred, only structural information will be recorded in the harmonic spectrum. However, in molecular ions electronic excitations often lie within a couple of electron volts from the ground state, leading to sizeable population amplitudes even for exponential scaling of ionization rates. The geometry of the molecular orbitals is also crucial ${ }^{19,20}$ and can further increase relative populations of the excited electronic states of the ion (tunnelling from deeper orbitals in the Hartree-Fock picture). The importance of different orbitals in harmonic generation has been pointed out in the experiments ${ }^{10,11}$.

Using the example of a $\mathrm{CO}_{2}$ molecule, we identify participating orbitals by measuring the phase of the high harmonics using two-slit interferometry ${ }^{21}$. In one arm we place randomly aligned molecules (a reference) and in the other, molecules aligned at a specific angle $\theta$.
Combined with amplitude measurements, the phase of the harmonic emission can give a 'fingerprint' of the molecular orbital. We identify at least three orbitals, and three states of the ion spanning a $4.3 \mathrm{eV}$ range of ionization potentials, that contribute to harmonic emission in $\mathrm{CO}_{2}$ molecules. Varying the laser intensity and controlling molecular alignment relative to laser polarization allows us to vary the contribution of each orbital to a given harmonic.

Access to multiple electronic states of the ion implies the potential for inducing and imaging multi-electron dynamics in the ion. We use high harmonic interferometry to reconstruct the shape and the motion of the hole created in the molecule upon ionization, gaining insight into attosecond dynamics of electron rearrangement during tunnelling.

There are two fundamental steps in resolving multi-electron dynamics: the identification of multiple orbitals (ionic states) participating in the process and the observation of their interference.

\section{Guidelines for tracking multiple orbitals}

When an intense laser pulse ionizes a molecule, its wavefunction splits into two components: a free electron wavepacket and its associated ion, and the neutral molecule. The laser field pushes the liberated electron away from the ion and then drives it back ${ }^{22}$. There, the electron can recombine with the ion, re-forming the neutral molecule in its original state and producing coherent radiation-high harmonics of the incident light. Different states of the molecular ion between ionization and recombination provide different 'channels' for this process (Fig. 1).

For each channel, specific electronic structure is encoded onto the harmonic amplitude and phase. These channels are different pathways that take the system from the same initial to the same final state via different states of the ion. They provide a basis for recording multielectron dynamics, including dynamics of electron rearrangement upon ionization. Their interference records the relative phases between the channels by mapping them onto the amplitude modulation in the harmonic spectra. The phases between the channels, 

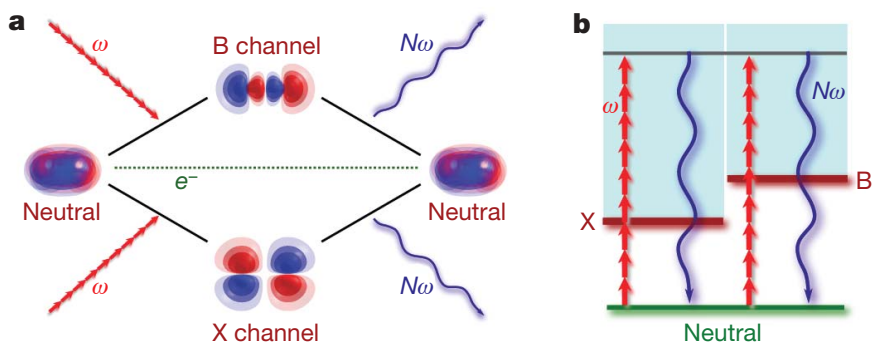

Figure 1 | Pictorial schemes of multichannel interference in high harmonic generation, for two channels. The orbitals sketched in a correspond to the two relevant channels $\mathrm{X}$ and $\mathrm{B}$ in a $\mathrm{CO}_{2}$ molecule aligned parallel to the laser polarization. Channels $\mathrm{X}$ and $\mathrm{B}$ are associated with $\widetilde{X}^{2} \Pi_{g}$ and $\tilde{B}^{2} \Sigma^{+}{ }_{u}$ states of the cation (HOMO and HOMO - 2 orbitals in the Hartree-Fock picture). Panel $\mathbf{b}$ illustrates channel interference in terms of a conventional nonlinear optics diagram.

accumulated between ionization and recombination, encode the underlying electronic dynamics with attosecond time resolution. High time resolution arises from the fact that different harmonics are emitted at different time-delays between ionization and recombination, within a fraction of a laser cycle $\mathrm{C}^{6,7,23,24}$. Each harmonic provides a 'frame' for the attosecond 'movie' that lasts about half a laser cycle (here, about 1.4 fsec.)

High harmonic generation begins with strong-field ionization and ends with recombination. Orbitals relevant for ionization and recombination are the Dyson orbitals (see, for example, ref. 25): the overlaps between the multi-electron wavefunctions of the neutral and the ion. Figure $2 \mathrm{a}$ shows the Dyson orbitals corresponding to creating the ion $\mathrm{CO}_{2}^{+}$in the ground state $\tilde{X}^{2} \Pi_{g}$ (channel X), first excited state $\tilde{A}^{2} \Pi_{u}$ (channel A), and the second excited state $\widetilde{B}^{2} \Sigma^{+}{ }_{u}$ (channel B). In the molecular orbital picture, these channels describe the removal of an electron from HOMO, HOMO - 1 and HOMO -2 , correspondingly.

Consider the Dyson orbital for channel X (Fig. 2a). Ionization along or perpendicular to the molecular axis will be suppressed ${ }^{19,20}$ owing to the destructive interference of currents from the orbital 'lobes' with opposite signs. This expectation is confirmed by the experiment ${ }^{26}$ and our calculations (see 'Supplementary Information'). For channel A, the Dyson orbital favours ionization perpendicular to the molecular axis. For channel B, it favours ionization parallel to the molecular axis. Thus, rotating the molecule relative to the laser polarization controls the relative contributions of different orbitals. The importance of excited states is not specific to $\mathrm{CO}_{2}$ : they will be involved whenever the HOMO has nodal planes and/or when electronic excitations in the ion are close in energy.

Now, consider recombination. The contribution of each channel $j$ with ionization potential $I_{\mathrm{p}, \mathrm{j}}$ to the $N$ th harmonic with energy $N \hbar \omega$ is proportional to the recombination matrix elements $d$ at the electron energy $E_{\mathrm{e}, j}=N \hbar \omega-I_{\mathrm{p}, j}$. These are shown in Fig. 2b-d (see Supplementary Information for details). As a function of the alignment angle $\theta$, both amplitudes and phases of $d$ vary in opposite directions for HOMO versus HOMO - 2. The overall minimum in $|d|^{2}$ for channel $X(\mathrm{HOMO})$ at small $\theta$ is not accidental: the same nodal plane in the Dyson orbital that suppresses ionization also suppresses recombination. These suppressions in channel $X$ help channel B to make a comparable contribution to harmonic emission for small angles $\theta$. Thus, measurement of the harmonic phases and amplitudes as a function of $\theta$ provides a diagnostic for the contribution of different orbitals.

The cutoff in the harmonic spectrum is determined by the maximum energy of the recombining electron plus the ionization potential $I_{\mathrm{p}}$ of the state into which it recombines ${ }^{27}$. Thus, contributions
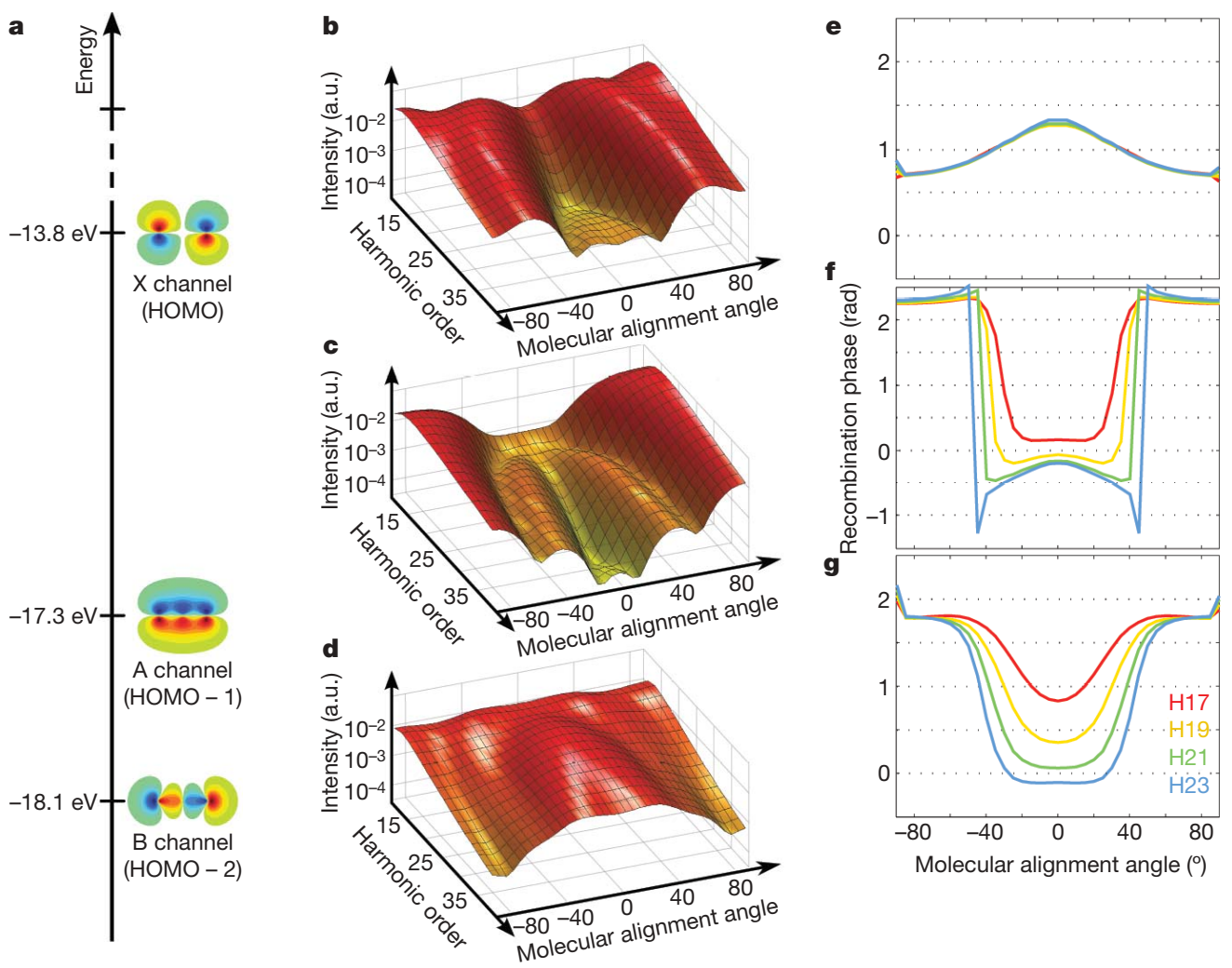

Figure 2 Contributions of different molecular orbitals to the harmonic emission. a, Dyson orbitals corresponding to different final states of the $\mathrm{CO}_{2}{ }^{+}$ion and the corresponding ionization energies. Differently coloured (blue and orange) orbital 'lobes' have opposite signs. b-g, Recombination amplitude squared (b-d) and recombination phase (e-g) show the calculated recombination matrix elements versus harmonic order and molecular alignment angle. The recombination phase (radians) is shown for selected harmonics H17, H19, H21 and H23. a.u., arbitrary units. 
from deeper orbitals with higher $I_{\mathrm{p}}$ can be studied by looking beyond the cutoff of the harmonics associated with the HOMO: the $\mathrm{B}$ channel in $\mathrm{CO}_{2}$ dominates the cutoff emission for molecules aligned at small angles $\theta$, while the A channel is significant for perpendicular alignment, $\theta \approx 90^{\circ}$.

\section{Setup for high-harmonic interferometry}

The main method of probing inner orbitals is to perform full characterization of the harmonic radiation, measuring both spectral phase and amplitude. To measure the dependence of the harmonic phase on the molecular alignment angle $\theta$ we use an approach pioneered by ref. 21 . We create two spatially separated harmonic sources in a gas jet (Fig. 3a). One is a reference with randomly aligned molecules, while in the other molecular alignment is controlled. In the far field, we observe an interference pattern, similar to the one obtained with Young's slits ${ }^{9,21,28,29}$. To observe a stable interference pattern, the fluctuations of delay between the two generating laser beams must be smaller than the harmonic period (for example, 130 attoseconds for the 21st harmonic of a 800 -nm laser pulse). We achieve a 1-attosecond stability by using a collinear scheme in which the two arms of the interferometer propagate together.

The position of the interference fringes is determined by the relative phase between the two sources. As the molecule is rotated away from the laser polarization, we observe a continuous shift of the fringes (Fig. 3b). Similar measurements for the $\mathrm{N}_{2}$ molecules show the shift in the opposite direction, proving the ability of our setup to measure both the magnitude and the sign of the harmonic phase.

\section{Identifying participating channels}

The measured harmonic amplitude and phase versus alignment angle $\theta$ are shown in Fig. $3 c$, d. The phase variation is $0.4 \mathrm{rad}$ for $\mathrm{H} 17$ and $\mathrm{H} 19$ and increases to $1.5 \mathrm{rad}$ for $\mathrm{H} 21$ and $2 \mathrm{rad}$ for $\mathrm{H} 23$. The curvature of the measured phase for $\mathrm{H} 23$ (Fig. 3d) is opposite to that calculated for the recombination matrix element in the $\mathrm{X}$ channel (HOMO) but similar to that for the B channel (Fig. 2e, g). This observation suggests that the harmonic generation in $\mathrm{CO}_{2}$ cannot be described assuming that only HOMO contributes to the process. The presence of the $\mathrm{B}$ channel is natural: at the laser intensity $I \approx 0.8 \times 10^{14} \mathrm{~W} \mathrm{~cm}^{-2}$ used in these measurements $\mathrm{H} 23$ is already beyond the cutoff for the $\mathrm{X}$ channel.

Turning to the measured amplitudes (Fig. 3c), for harmonics 17 and 19 the signal is minimum at parallel alignment and maximum around $60^{\circ}$. Harmonics 23 and 25 (latter not shown) are maximal at parallel alignment. The former behaviour is consistent with the recombination matrix elements for the $\mathrm{X}$ channel, while the latter is consistent with the B channel (see Fig. 2). We note that for the $\mathrm{X}$ channel, both ionization and recombination are strongly suppressed at parallel alignment, while the observed minimum in the harmonic emission is rather modest. Therefore, experiment and theory indicate that the B channel (HOMO-2 orbital) is also contributing to the harmonics below $\mathrm{H} 23$ at this laser intensity. In this region the harmonic emission results from the interference of the contributions from different channels, as described in Fig. 1.

\section{Channel interference and hole dynamics}

We now turn to the analysis of the channel interference and the multi-electron dynamics it encodes. We show that this dynamics can be decoded by studying the interference pattern in the harmonic spectrum as a function of the laser intensity.

Experimentally, we have recorded the harmonic spectra in $\mathrm{CO}_{2}$ molecules as a function of molecular alignment angle, for laser intensities from $I \approx 5 \times 10^{13} \mathrm{~W} \mathrm{~cm}^{-2}$ to $I \approx 2 \times 10^{14} \mathrm{~W} \mathrm{~cm}^{-2}$ (Fig. $4 \mathrm{a}, \mathrm{b}$ and Fig. 5b, c). There is a clear minimum in the harmonic spectrum at $\theta=0^{\circ}$ (Fig. $4 \mathrm{a}, \mathrm{b}$ ), which shifts to higher orders with increasing intensity, from $\mathrm{H} 19$ at $0.55 \times 10^{14} \mathrm{~W} \mathrm{~cm}^{-2}$ to $\mathrm{H} 29$ at $1.8 \times 10^{14} \mathrm{~W} \mathrm{~cm}^{-2}$ (Fig. 5c). This observation explains why the minimum has been observed at vastly different positions in previous experiments ${ }^{4,5,8,9}$. The minimum has previously been associated with the structure of HOMO, but its mobility with intensity indicates that this picture is incomplete. Indeed, up to the details of the molecular alignment and saturation of ionization ${ }^{17}$, the structural features are tied to the electron de Broglie wavelength and hence to a specific

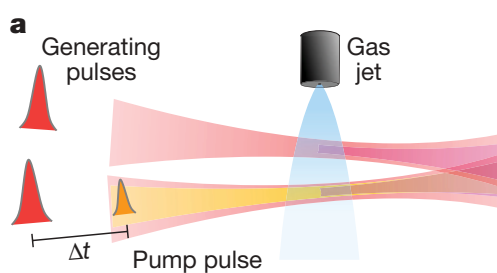

c

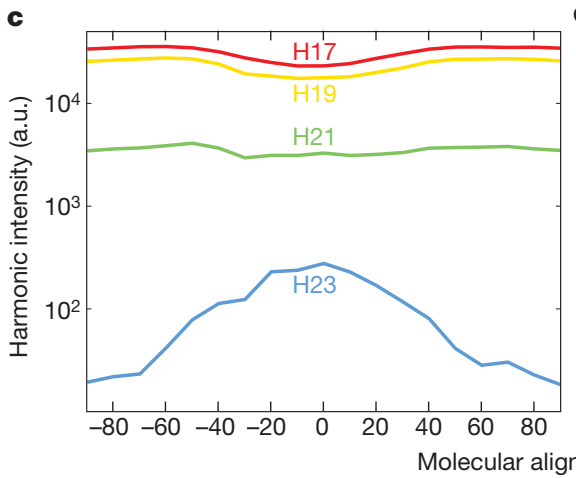

3 Harmonic phase interferometry. a, Experimental setup. Two harmonic sources are created by focusing two synchronized non-collinear laser beams (probe) in a gas jet. A third laser beam (pump) is superimposed on the lower source to control molecular alignment. The pump-probe delay $\Delta t$ is adjusted so that molecules are aligned along the pump pulse polarization when the generating pulses hit the jet. The harmonic radiation is analysed by an extreme ultraviolet (XUV) spectrometer that images the interference pattern of each harmonic. $\mathbf{b}$, Measured interference fringes as a
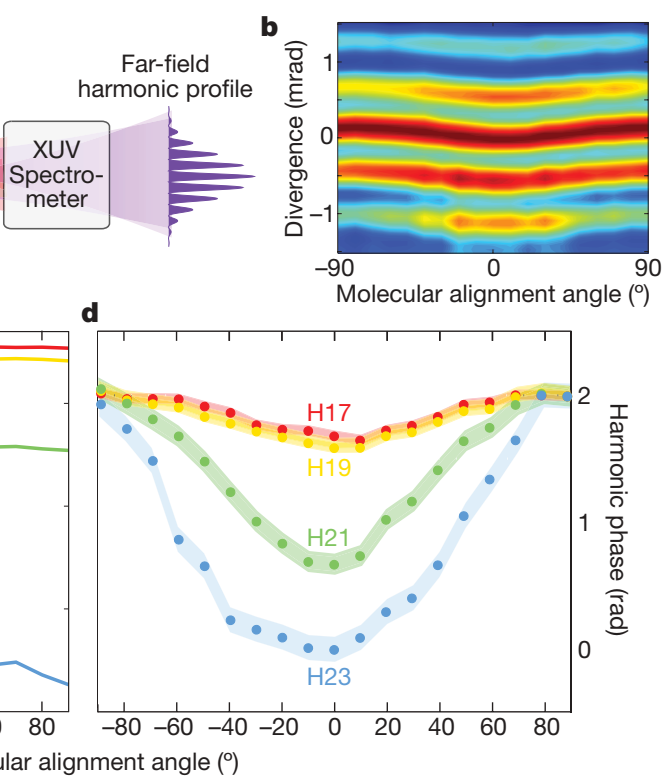

function of molecular angle for the 21st harmonic in $\mathrm{CO}_{2}$. The fringe pattern for each angle is averaged over 5,000 laser shots and normalized.

c, d, Measured harmonic intensities (c) and phases (d) at

$I \approx 8 \times 10^{13} \mathrm{~W} \mathrm{~cm}^{-2}$. The phases of different harmonics are vertically shifted to coincide at $90^{\circ}$. The thickness of the line is the error bar, given by the standard deviation of the phase fluctuations in the absence of the pump beam (0.04 rad for $\mathrm{H17})$. 

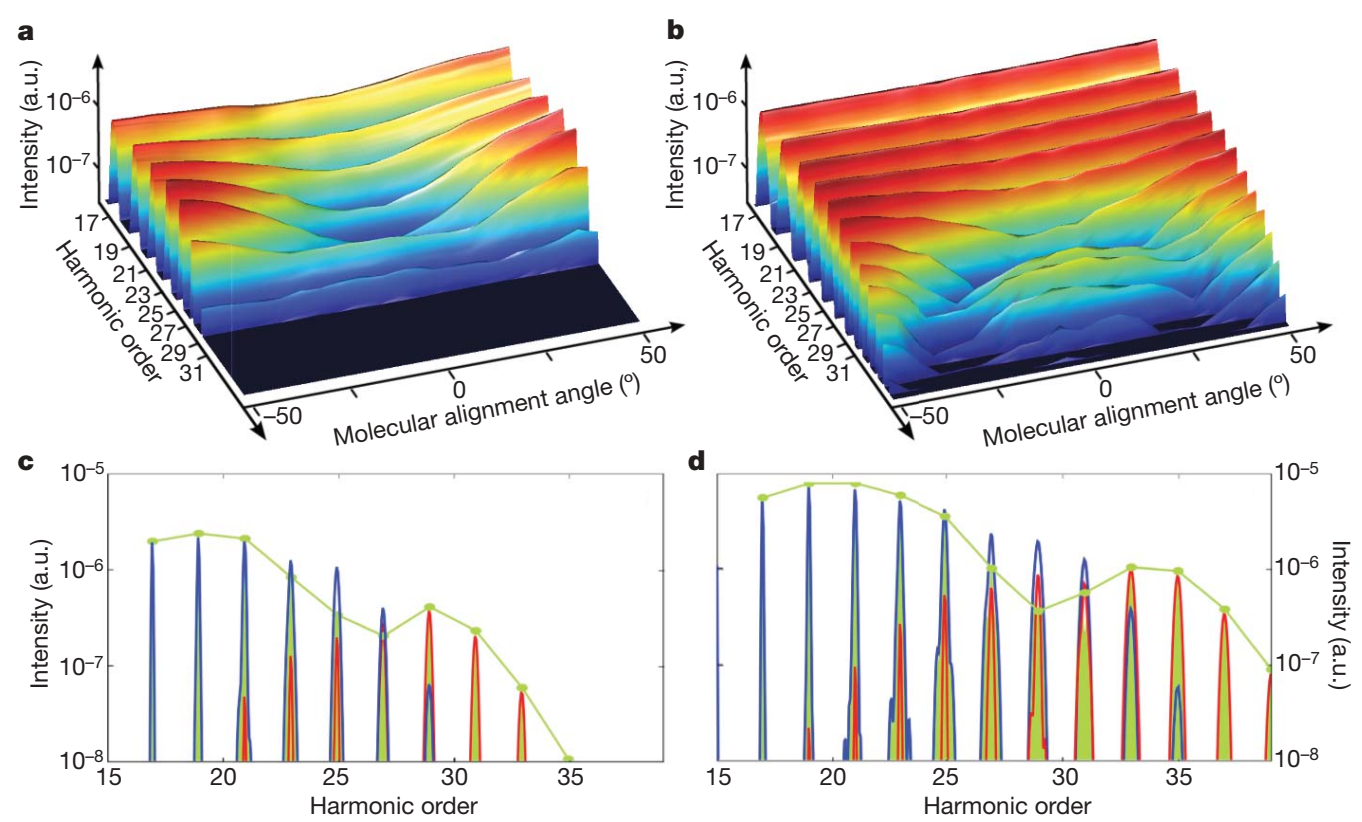

Figure 4 | Harmonic spectra in $\mathrm{CO}_{2}$ molecules. a, b, Measured harmonic spectra as a function of the harmonic number and the molecular angle in $\mathrm{CO}_{2}$, for $I \approx 1.1 \times 10^{14} \mathrm{~W} \mathrm{~cm}^{-2}$ (a) and $I \approx 1.8 \times 10^{14} \mathrm{~W} \mathrm{~cm}^{-2}$

(b). c, d, Calculated spectra for $\theta=0^{\circ}$ (after averaging over alignment distribution in the molecular ensemble), for $I=1.26 \times 10^{14} \mathrm{~W} \mathrm{~cm}^{-2}(\mathrm{c})$ and

$I=1.72 \times 10^{14} \mathrm{~W} \mathrm{~cm}^{-2}(\mathrm{~d})$. The blue and red lines are the contributions from the channel X (HOMO) and channel B (HOMO - 2). The green line and the shaded spectrum is the total harmonic spectrum resulting from the coherent superposition of these contributions.
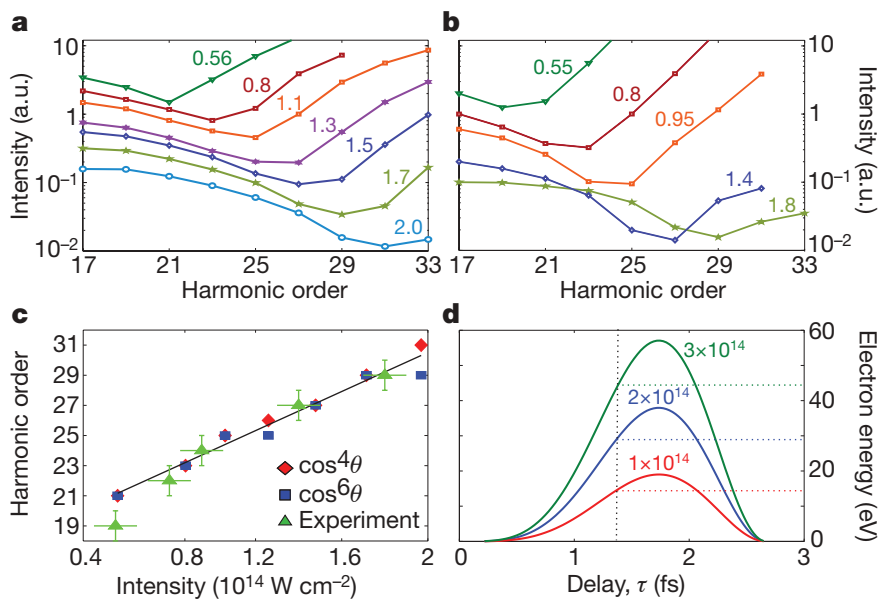

e

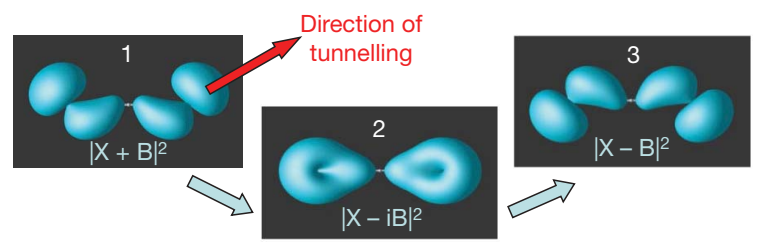

Figure 5 | Reconstruction of the hole dynamics. Calculated (a) and measured (b) spectra (integrated over each harmonic line) for different intensities and $\theta=0^{\circ}$ (shifted vertically for better visibility). Intensities are given in units of $10^{14} \mathrm{~W} \mathrm{~cm}{ }^{-2}$. The spectra are normalized by the signal at $\theta=50^{\circ}$. c, Measured (triangles) and calculated intensity dependence of the position of the minimum. Calculations are for $\cos ^{4} \theta$ (diamonds) and $\cos ^{6} \theta$ (squares) alignment distributions. Error bars in the position of the minimum are based on standard deviation in the fluctuations of harmonic intensity. The line is a least-squares fit to the calculations. d, Origin of the intensity dependence for a dynamical minimum. For fixed emission time (horizontal axis) the electron energy $E_{\mathrm{e}}$ (vertical axis) and the harmonic order $N \hbar \omega=E_{\mathrm{e}}+I_{\mathrm{p}}$ linearly depend on the intensity $\left(\right.$ red, $I=10^{14} \mathrm{~W} \mathrm{~cm}^{-2}$; blue, $I=2 \times 10^{14} \mathrm{~W} \mathrm{~cm}^{-2}$; green, $\left.I=3 \times 10^{14} \mathrm{~W} \mathrm{~cm}^{-2}\right)$. e, Visualization of the hole dynamics: (1) initial shape of the hole, (2) hole density quarterperiod and (3) a half-period later.

harmonic order. The results of the previous section suggest that we are observing the destructive interference of channels X (HOMO) and $\mathrm{B}$ $(\mathrm{HOMO}-2)$.

Our conclusion is supported by Fig. $4 \mathrm{c}$ and d, which shows the calculated spectra at $\theta=0^{\circ}$. The calculations (see Methods and Supplementary Information sections) include the contribution of $\mathrm{X}, \mathrm{A}$ and $\mathrm{B}$ channels. To simulate experimental conditions, the contribution of the 'long trajectories' has been filtered out. Emitted radiation was coherently averaged over alignment distributions with parameters typical for the experiments. The $\mathrm{X}$ channel (blue curve) dominates the emission for lower harmonics, while the $\mathrm{B}$ channel (red curve) takes over for the higher harmonics. The total spectrum (green curve) records the relative phase between the channels by mapping it into the amplitude modulations. The minimum corresponds to the destructive interference between the channels.

The position of the minimum versus the laser intensity is shown in Fig. 5. Both theoretical (Fig. 5a) and experimental (Fig. 5b) spectra have been normalized to those for $\theta=50^{\circ}$. At this angle, channel X dominates the spectrum, channel interference is negligible, and the spectrum has no pronounced minima or maxima. Normalization eliminates the instrument response effects and makes the quantitative comparison of theory and experiment possible. Experiment and theory are in good agreement.

Why does the minimum shift with intensity and what can we learn from it? There are four different sources of relative phase between the channels, which control their interference (see equation (1)): (1) the recombination phase (Fig. 2e-g); (2) the continuum evolution phase, which is dominated by the strong laser field and is similar for different channels (see 'Supplementary Information'); (3) the evolution of the electronic states $j$ of the ion $\Psi_{j}(t) \propto e^{-i E_{j} \tau}$ during the time-interval $\tau$ between ionization and recombination $\left(E_{j}\right.$ are the state energies); and (4) the ionization phase (see below).

The interference minimum is tied to that time-delay $\tau^{*}$ when channels interfere destructively. For a given $\tau^{*}$, the energy of the continuum electron $E_{\mathrm{e}}\left(\tau^{*}\right)$ varies linearly with the laser intensity (Fig. $5 \mathrm{~d}$ ). Therefore, in first approximation, the minimum should move linearly with intensity. A weaker influence of the laser field on the bound 
electrons in the $\mathrm{CO}_{2}{ }^{+}$ion is also included in our calculations (see Supplementary Information).

Experiment confirms that the harmonic minimum shifts approximately linearly with intensity. A least-squares fit to the data (Fig. 5c) shows that the position of the minimum scales as $N \hbar \omega \propto$ $(1.7 \pm 0.1) U_{p}$, where $U_{p}$ is the mean quiver energy of the electron in the laser field. The mapping of the harmonic numbers onto the time-delay $\tau$ between ionization and recombination ${ }^{6,7,23,24,30}$, first used to track the motion of protons ${ }^{6,7,24}$, yields $\tau^{*}=1.17 \pm 0.1 \mathrm{fsec}$ after ionization. At this moment the relative phase accumulated in the ion is $\left(E_{\mathrm{B}}-E_{\mathrm{X}}\right) \tau^{*} \approx(2.44 \pm 0.2) \pi$. Destructive interference requires a total phase difference of $3 \pi$. The phase between the channels also includes the relative phase between the recombination matrix elements, which is about $0.5 \pi$ (note the opposite parity of $\mathrm{HOMO}$ and HOMO -2 ). Thus, the total relative phase between the channels is indeed $3 \pi$ at $\tau^{*}$, demonstrating the consistency of our reconstruction. Additionally, reconstruction of $\tau^{*}$ from the position of the minimum for each intensity separately yields the same result.

Coherent population of two ionic states (for example, $\mathrm{X}$ and $\mathrm{B}$ ) implies excitation of the bound electronic wavepacket in the ion. Interference between the channels records the wavepacket dynamics. This two-state dynamics has one non-trivial parameter-the initial relative phase $\varphi$ between the two ionic states that could have been acquired during ionization. In the static tunnelling limit, in each channel the wavefunction after electron tunnelling through the barrier is an extension of the real wavefunction that characterizes the bound state, implying $\varphi=0$. Non-zero $\varphi$ would reflect nonadiabatic dynamics of electron rearrangement during strong-field ionization. We note that $\varphi$ is different from the phase of the dipole transition associated with recombination. Indeed, recombination is a single-photon transition, whereas optical tunnelling proceeds via absorption of many infrared photons.

Knowing the instant of destructive interference allows us to find $\varphi$. At high intensities, the reconstructed value $\tau^{*}=1.17 \pm 0.1 \mathrm{fsec}$ is consistent with $\varphi=0$, expected in the tunnelling regime. Changing $\varphi$ to $\varphi= \pm \pi / 2$ in the theoretical calculations moves the calculated positions of the minimum beyond the experimental error; see Supplementary Information.

For the same state of the continuum electron, the initial phase between the channels determines the initial location/shape of the hole left in the molecule by ionization. It could be visualized as a superposition of the Dyson orbitals $\Psi^{(\mathrm{D})}{ }_{\mathrm{X}}$ and $\Psi^{(\mathrm{D})}{ }_{\mathrm{B}}$ corresponding to channels X and B: $\left|\Psi_{\text {init }}\right|^{2}=\left|\Psi^{(\mathrm{D})} \mathrm{X}+e^{+i \varphi} \Psi^{(\mathrm{D})}{ }_{\mathrm{B}}\right|^{2}$, for $\varphi=0$ (see Fig. $5 \mathrm{e}$, snapshot 1 ). The phase $\varphi=0$ yields the maximum possible extension of the density in the direction of tunnelling (for the two channels X and B.) Snapshots 2 and 3 in Fig. 5e show the hole density quarter-period $\left(\left|\Psi^{(\mathrm{D})}{ }_{\mathrm{X}}-i \Psi^{(\mathrm{D})}{ }_{\mathrm{B}}\right|^{2}\right)$ and half-period later $\left(\left|\Psi^{(\mathrm{D})} \mathrm{X}-\Psi_{\mathrm{B}}^{(\mathrm{D})}\right|^{2}\right)$.

\section{Perspectives}

Our work shows how measurements of the phases and amplitudes of high harmonic radiation can be used to follow not only vibrational ${ }^{6,7}$, but also multi-electron dynamics in molecules with attosecond temporal resolution. These dynamics can be induced by ionization, as in the $\mathrm{CO}_{2}$ molecule. Multi-electron excitations can also be induced by the laser field between ionization and recombination, on the subcycle timescale ${ }^{31-33}$. Multiple ionic states are naturally excited during photo-ionization and/or non-adiabatic transitions induced by nuclear motion. High harmonic emission, used as a probe in ultrafast pump-probe experiments (see, for example, refs 10 and 34), provides a new tool with which to measure the underlying dynamics.

The question of the relative phase $\varphi$ between different channels in strong-field ionization has not been addressed before. This phase records interactions between the departing electron and the electrons of the core, that is, the coupling between different channels during strong-field ionization. We expect that the phase $\varphi$ will depend on the ionization regime, because the coupling between the channels during strong-field ionization is caused not noly by electron-electron interaction, but also laser field. Analysis of this dependence based purely on the experimental measurements would require a separate characterization of the phase of recombination, which should be possible using interferometric techniques of attosecond photoelectron spectroscopy (see for example, refs 35 and 36). Our experiment (Fig. 5c) seems to suggest that in the multiphoton regime (below $I \approx 1.1-1.2 \times 10^{14} \mathrm{~W} \mathrm{~cm}^{-2}$ ), the intensity dependence of the minimum might be deviating from the linear; see Supplementary Information for discussion. However, the deviation is at the limits of the accuracy of our measurement. Thus, high-harmonic interferometry offers a versatile approach to observing a wide variety of attosecond processes. Combined with the recently developed perturbative methods for attosecond metrology ${ }^{30}$ and the measurements of the polarization state ${ }^{37}$, it will greatly facilitate a new frontier of attosecond dynamics measurements.

\section{METHODS SUMMARY}

Experimentally, we use a 50-Hz Ti:Sa laser system providing 15-mJ 30-fs pulses at $800 \mathrm{~nm}$. The laser pulse is split by a polarizing beam splitter and a half-waveplate. The transmitted beam is the alignment beam. Its polarization direction is rotated with a second half-waveplate. Critical to the experiment is a very stable interferometer that is formed by splitting the reflected beam into two generating beams. We exploit the refractive index difference between two axes of a birefringent crystal to produce the two beams, separated by a small angle. Both beams share all optical elements. The three beams are focused by a $50-\mathrm{cm}$ lens in a $1-\mathrm{mm}$-long pulsed gas jet. The high harmonics produced by the two generating beams, separated by $\sim 70 \mu \mathrm{m}$, overlap in the far field, which serves as a beam combiner. The harmonic intensity is obtained by integrating the far-field spatial profile, while the harmonic phase is extracted by means of the Fourier transform.

Theoretically, multichannel harmonic emission is described by the induced dipole:

$$
\mathbf{D}(t) \approx\left\langle\Psi_{\mathrm{NT}}(t)|\hat{\mathbf{d}}| \sum_{j} \hat{\mathbf{A}} \Psi_{j}(t) \chi_{\mathrm{C}, j}(t)\right\rangle+c . c .
$$

Here $\Psi_{\mathrm{NT}}(t)$ describes the evolution of the neutral molecule during the laser pulse, including depletion by ionization, while the electron-ion wavepacket created by ionization is $\Psi_{\mathrm{ei}}(t)=\sum_{j} \hat{\mathbf{A}} \Psi_{j}(t) \chi_{\mathrm{C}, j}(t)$. Index $j$ labels the channels

involving different ionic states, $\Psi_{j}(t)$ describes their evolution between ionization and recombination, $\chi_{\mathrm{C}, j}(t)$ are the continuum wavepackets correlated to these ionic states, the operator $\hat{\mathbf{A}}$ anti-symmetrizes the electrons, and c.c. indicates the complex conjugate. The field of Nth harmonic is determined by the Fourier transform $\tilde{\mathbf{D}}(N \omega)$ of $\mathbf{D}(t)$ at the frequency $N \omega$. The Fourier transform enforces the energy conservation law, $N \hbar \omega=E_{\mathrm{e}}+I_{\mathrm{p}, j}$, where $E_{\mathrm{e}}$ is the energy of the returning electron and $I_{\mathrm{p}, j}$ is the ionization potential in channel $j$.

\section{Received 20 May 2008; accepted 3 July 2009.}

Published online 22 July 2009.

1. Cohen, H. D. \& Fano, U. Interference in the photo-ionization of molecules. Phys. Rev. 150, 30-33 (1966).

2. Lein, M. et al. Role of the intermolecular phase in high harmonic generation. Phys. Rev. Lett. 88, 183903 (2002).

3. Itatani, J. et al. Tomographic imaging of molecular orbitals. Nature 432, 867-871 (2004).

4. Kanai, T., Minemoto, S. \& Sakai, H. Quantum interference during high-order harmonic generation from aligned molecules. Nature 435, 470-474 (2005).

5. Vozzi, C. et al. Controlling two-center interference in molecular high harmonic generation. Phys. Rev. Lett. 95, 153902 (2005).

6. Baker, S. et al. Probing proton dynamics in molecules on an attosecond time scale. Science 312, 424-427 (2006).

7. Baker, S. et al. Dynamic two-center interference in high-order harmonic generation from molecules with attosecond nuclear motion. Phys. Rev. Lett. 101, 053901 (2008).

8. Boutu, W. et al. Coherent control of attosecond emission from aligned molecules Nature Phys. 4, 545-549 (2008).

9. Zhou, X. et al. Molecular recollision interferometry in high harmonic generation. Phys. Rev. Lett. 100, 073902 (2008).

10. $\mathrm{Li}, \mathrm{W}$. et al. Time-resolved dynamics in $\mathrm{N}_{2} \mathrm{O}_{4}$ probed using high harmonic generation. Science 322, 1207-1211 (2006).

11. McFarland, B. K. et al. High harmonic generation from multiple orbitals in $\mathrm{N}_{2}$. Science 322, 1232-1235 (2006).

12. Rosca-Pruna, F. \& Vrakking, M. J. J. Experimental observation of revival structures in picosecond laser-induced alignment of $I_{2}$. Phys. Rev. Lett. 87, 153902 (2001). 
13. Stapelfeldt, H. \& Seideman, T. Aligning molecules with strong laser pulses. Rev, Mod. Phys. 75, 543-557 (2003).

14. Le, A. T., Morishita, T. \& Lin, C. D. Extraction of the species-dependent dipole amplitude and phase from high-order harmonic spectra in rare-gas atoms. Phys. Rev. A 78, 023814 (2008).

15. Morishita, T., Le, A. T., Chen, Z.\& Lin, C. D. Accurate retrieval of structural information from laser-induced photoelectron and high-order harmonic spectra by few-cycle laser pulses. Phys. Rev. Lett. 100, 013903 (2008).

16. Le, V. H., Le, A. T., Xie, R. H. \& Lin, C. D. Theoretical analysis of dynamic chemical imaging with lasers using high-order harmonic generation. Phys. Rev. A 76, 013414 (2007).

17. Le, A. T., Tong, X. M. \& Lin, C. D. Evidence of two-center interference in high-order harmonic generation from $\mathrm{CO}_{2}$. Phys. Rev. A 73, 041402 (2006).

18. Lein, M. Molecular imaging using recolliding electrons. J. Phys. B 40, R135-R173 (2007).

19. Tong, X. M., Zhao, Z. X. \& Lin, C. D. Theory of molecular tunneling ionization. Phys. Rev. Lett. 66, 033402 (2002).

20. Muth-Böhm, J., Becker, A. \& Faisal, F. H. M. Suppressed molecular ionization for a class of diatomics in intense femtosecond laser fields. Phys. Rev. Lett. 85, 2280-2283 (2000).

21. Zerne, R. et al. Phase locked high-order harmonic emission. Phys. Rev. Lett. 79, 1006-1009 (1997).

22. Corkum, P. B. Plasma perspective on strong field multiphoton ionization. Phys. Rev. Lett. 71, 1994-1997 (1993).

23. Mairesse, $Y$. et al. Attosecond synchronization of high-harmonic soft X-rays. Science 302, 1540-1543 (2003).

24. Lein, M. Attosecond probing of vibrational dynamics with high-harmonic generation. Phys. Rev. Lett. 94, 053004 (2005)

25. Ortiz, J. V. Quasiparticle approximations and electron propagator theory. Int. J. Quantum Chem. 95, 593-599 (2003).

26. Pavicic, D. et al. Direct measurement of the angular dependence of ionization for $\mathrm{N}_{2}, \mathrm{O}_{2}$, and $\mathrm{CO}_{2}$ in intense laser fields. Phys. Rev. Lett. 98, 243001 (2007).

27. Lewenstein, $M$. et al. Theory of high harmonic generation by low-frequency laser fields. Phys. Rev. A 49, 2117-2132 (1994).
28. Descamps, D. et al. Extreme ultraviolet interferometry measurements with high order harmonics. Opt. Lett. 25, 135-137 (2000).

29. Corsi, C., Pirri, A., Sali, E., Tortora, A. \& Bellini, M. Direct interferometric measurement of the atomic dipole phase in high-order harmonic generation. Phys. Rev. Lett. 97, 023901 (2006).

30. Dudovich, N. et al. Measuring and controlling the birth of attosecond XUV pulses. Nature Phys. 2, 781-786 (2006).

31. Lezius, M. et al. Nonadiabatic multielectron dynamics in strong field molecular ionization. Phys. Rev, Lett. 86, 51-89 (2001)

32. Markevitch, A. N. et al. Nonadiabatic dynamics of polyatomic molecules and ions in strong laser fields. Phys. Rev. A 68, 011402 (2003).

33. Litvinyuk, I. et al. Shakeup excitation during optical tunnel ionization. Phys. Rev. Lett. 94, 033003 (2005).

34. Wagner, N. et al. Monitoring molecular dynamics using coherent electrons from high-harmonic generation. Proc. Natl Acad. Sci. USA 103, 13279-13285 (2006).

35. Remetter, T. et al. Attosecond electron wave packet interferometry. Nature Phys. 2, 323-326 (2006).

36. Corkum, P. B. \& Krausz, F. Attosecond science. Nature Phys. 3, 381-387 (2007).

37. Levesque, J. et al. Polarization state of high-order harmonic emission from aligned molecules. Phys. Rev. Lett. 99, 243001 (2007).

Supplementary Information is linked to the online version of the paper at www.nature.com/nature.

Acknowledgements We appreciate discussions with A. Stolow and M. Spanner. This research was partially supported by the NSERC SRO and an AFOSR grant. O.S. acknowledges the Leibniz SAW award, and M.I. acknowledges support from the Alexander von Humboldt foundation.

Author Contributions O.S., S.P. and M.Y.I. developed theory, and Y.M., N.D., D.V. and P.C. performed experiments. All authors contributed to the analysis of data and writing the paper.

Author Information Reprints and permissions information is available at www.nature.com/reprints. Correspondence and requests for materials should be addressed to O.S. (olga.smirnova@mbi-berlin.de). 


\section{SUPPLEMENTARY INFORMATION}

Supplementary Material is organized as follows. Sections I,II and Fig. 1 describe experimental details. Sections III-X and Fig. 2,3 describe details of the theoretical calculations. Sections XI-XIII and Fig. 3 discuss the accuracy of the reconstruction procedure used in the paper and the possible change of the ionization phase between tunnelling and multi-photon regimes. 


\section{HIGH HARMONIC GENERATION AND ANALYSIS}

The target is a pulsed gas jet with 3 atm backing pressure and jet density in the interaction zone of $\sim 10^{17} \mathrm{~cm}^{-3}$. In principle, an aligned molecular gas is a birefringent medium[2]. However, with our low density and length the effect of molecular alignment on the phase of the IR light is negligible.

High harmonics are analyzed by an XUV spectrometer composed of a grazing incidence concave grating with variable groove spacing. This spectrometer disperses the spectrum in the horizontal plane and lets the beam diverge in the vertical plane. The spectrally resolved far field harmonic profile is detected on microchannel plates associated to a phosphor screen and imaged by a CCD camera.

Two classes of electron trajectories contribute to the generation of the same harmonic. By adjusting jet position relative to the laser focus and selecting the central part of the harmonic spectrum[1], we isolate the contributions from the short electron trajectories.

\section{EXPERIMENTAL DETAILS ON TWO-SLIT INTERFEROMETRY}

We create the two interfering beams using a $750 \mu \mathrm{m}$ thick calcite plate. It splits the incoming beam in two orthogonally polarized delayed pulses. Since the plate is tilted with respect to the axis of the experiment, the two transmitted beams are vertically shifted by $\delta x$. The plate is inside a telescope constituted of a $f_{1}=150 \mathrm{~mm}$ convergent lens and a $f_{2}=-100 \mathrm{~mm}$ divergent one. At the exit of the divergent lens, the two delayed beams are not parallel. Their relative angle is $\delta x / f_{1}$. They are also time shifted with respected to each other. A second $750 \mu \mathrm{m}$ calcite plate, whose axes are crossed with respect to the first one, re-synchronizes the pulses. A half waveplate rotates the polarizations by 45 degrees, and a polarizer projects both polarizations on the same axis. A schematic of the beam-splitter is shown in Fig. 1.

The two generating beams and the alignment beam are focused into the gas jet by a $F=50 \mathrm{~cm}$ lens. All beams are focused in $\sim 100 \mu \mathrm{m}$ spots vertically separated by $\Delta x=$ $\delta x F / f_{1} \approx 70 \mu \mathrm{m}$. The intensity of the generating and alignment beams is respectively $I \sim 10^{14} \mathrm{~W} . \mathrm{cm}^{-2}$ and $I \sim 5 \times 10^{13} \mathrm{~W} . \mathrm{cm}^{-2}$. The alignment beam did not produce harmonics. We image the focal plane on a CCD camera and adjust the relative phase of the two beams 


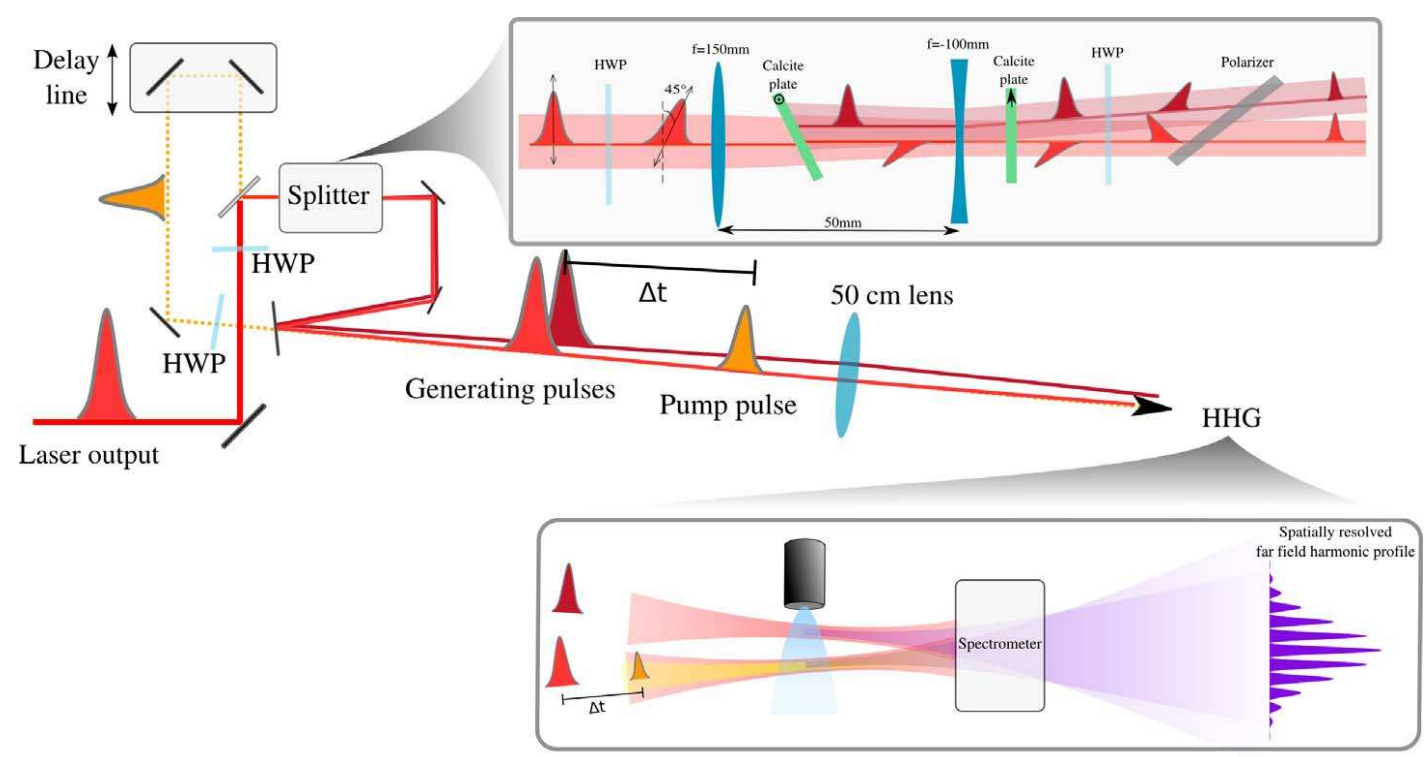

FIG. 1: Experimental setup for two source interferometry.

to achieve destructive interference in the region where they are overlapped. This imaging also allows us to overlap the focus of the alignment beam with the lower generating beam.

\section{GENERAL THEORETICAL MODEL}

Theoretically, we generalize [3] and approximate $\mathbf{D}(t)$ as:

$$
\mathbf{D}(t, \theta) \propto \sum_{j} a_{\text {ion, } j}\left[t_{b}, \Theta\right] a_{\text {prop }}\left[t, t_{b}, \theta\right]\left\langle\Psi_{N T}(t, \theta)|\hat{\mathbf{d}}| \hat{A} \Psi_{j} A_{j}(t, \theta) \chi_{C, j}(t, k(t), \theta)\right\rangle+c . c .
$$

This general expression provides a convenient framework, which allows one to go beyond such approximations as the single-active electron approximation and the strong-field approximation. The N-electron wavefunction $\Psi_{N T}$ describes the evolution of the neutral molecule during the laser pulse, including depletion by ionization. The amplitudes $A_{j}(t, \theta)$ describe laser induced dynamics in the ion in the basis of ionic states $\Psi_{j}$, starting in a state $j$ at the the moment of ionization $t_{b}=t_{b}(t)$, until recombination at $t$. The correlated continuum electron wavepackets $\chi_{C, j}(t)$ reflect the structure of the Dyson orbital corresponding to the 
ionization channel. The wavepackets $\chi_{C, j}(t)$ include two components. First is the scattering state $\phi_{C, j}(t, k(t), \theta)$, correlated to the state of the ion $\Psi_{j}$ and characterized by the (asymptotic) kinetic momentum $k(t)$ acquired from the laser field. Second is the channel amplitude, which includes ion-state-specific sub-cycle ionization amplitude $a_{\mathrm{ion}, \mathrm{j}}$ at the moment $t_{b}$ and the propagation amplitude $a_{\text {prop }}$ between $t_{b}$ and $t$. The harmonic amplitudes, phases and polarizations are calculated by using the Fourier transform of Eq.(1). Long trajectories are filtered out prior to the Fourier transform of $\mathbf{D}(t)$ using a smooth temporal filter. Thus, for each $t$ there is only one $t_{b}$ that contributes to the induced dipole $\mathbf{D}(t, \theta)$.

We use complete active space (CAS) self-consistent field (SCF) method to calculate the 22-electron wavefunction of $\mathrm{CO}_{2}$ and 21-electron wavefunctions of the ionic states $\tilde{X}, \tilde{A}, \tilde{B}$ of $\mathrm{CO}_{2}^{+}$. Laser-induced polarization of the neutral is described in the quasistatic approximation. We calculate the transition dipole moments between essential ionic states and use them to describe laser-induced dynamics in the ion between ionization and recombination within the $\tilde{X}, \tilde{A}, \tilde{B}$ states. Non-resonant Stark shift due to the coupling to other states is also included, and is calculated in the quasi-static approximation. Non-adiabatic transitions between the ionic states, between ionization and recombination, are naturally incorporated in the formalism but are negligible at $\lambda=800 \mathrm{~nm}$. Strong-field eikonal Volkov approximation [4] takes care of continuum dynamics, including channel-specific scattering on the parent ion during the recollision. Ionization is calculated using the method of $[5,6]$ to include the geometry of the Dyson orbital and Coulomb-corrected sub-cycle ionization rates from [7]. Further details are given below.

\section{IONIZATION AMPLITUDE.}

We use analytical approximation to the general approach developed by A. Becker and co-workers[6, 8-10]. Channel-specific ionization rates are calculated for the Dyson orbital $\left|\Psi_{D, j}\right\rangle=\sqrt{N}\left\langle\Psi_{j} \mid \Psi_{N T}\right\rangle$ corresponding to the channel $j$ ( $N$ is the number of electrons.) To take into account multi-center geometry of the Dyson orbital, it is written as a linear combination of spherical atomic orbitals. This representation allows us to factorize the ionization amplitude,

$$
a_{\mathrm{ion}, \mathrm{j}}\left[t_{b}, \theta\right]=a_{\mathrm{ion}, \mathrm{j}}^{(0)}\left[t_{b}\right] R_{j}(\theta)
$$


approximating it as a product of the 'atomic' ionization amplitude $a_{\mathrm{ion}}^{(0)}\left[t_{b}\right]$ from a single atomic orbital and an angular-dependent term $R(\theta)$, determined by the interference of the atomic orbitals.

The 'atomic' amplitude depends on the ionization potential of the orbital, i.e. on the transition energy between the ground state of the neutral and the final ionic state. This transition energy includes the relative Stark shift between the two states. The shift is calculated in the quasi-static approximation. To calculate the sub-optical cycle 'atomic' ionization amplitude, we use the Yudin-Ivanov formula [7], including the Coulomb corrections as described in $[7]$.

Interference of atomic orbitals yields angle-dependent factor $R(\theta)$, given by the Fourier transform of the Dyson orbital into the momentum space. The Fourier transform is first squared and then integrated with the tunnelling-induced momentum distribution for the outgoing electron, yielding $R^{2}(\theta)$. Our analytical approximation to $R(\theta)$ uses the method described in [5]: the momentum filter is Gaussian in the direction perpendicular to laser polarization, with the width determined by the Keldysh tunnelling time $\tau=\sqrt{2 I_{p}} / E$, where $E$ is the electric field amplitude for the given cycle of the multi-cycle laser pulse.

Results of our calculations are shown in Fig.2, which shows angular dependence of strongfield ionization for the three relevant channels, normalized to a single atomic orbital with the same ionization potential. The laser intensity is $I=1.7 \times 10^{14} \mathrm{~W} / \mathrm{cm}^{2}$ and wavelength $\lambda=800 \mathrm{~nm}$.

\section{MULTIELECTRON STATES AND DYNAMICS IN THE NEUTRAL AND THE ION.}

We use quantum chemistry approaches for bound electrons to find $\Psi_{N T}$ and $\Psi_{j}$. The 22-electron wave function for the ground state of $\mathrm{CO}_{2}$ and 21-electron wave functions for $\tilde{X}, \tilde{A}, \tilde{B}$ ionic states in $\mathrm{CO}_{2}^{+}$are calculated using complete active space self-consistent field method. For the three relevant electronic states of the ion, we correlate 15 electrons in 10 active orbitals, using correlation-consistent basis set of triple- $\zeta$ quality in the valence region (cc-pVTZ). Polarization of the states by the external laser field has been calculated in the quasi-static approximation. The difference between the Stark shifts of the neutral and ionic ground states remains small even at high intensities, reaching about $0.5 \mathrm{eV}$ at 


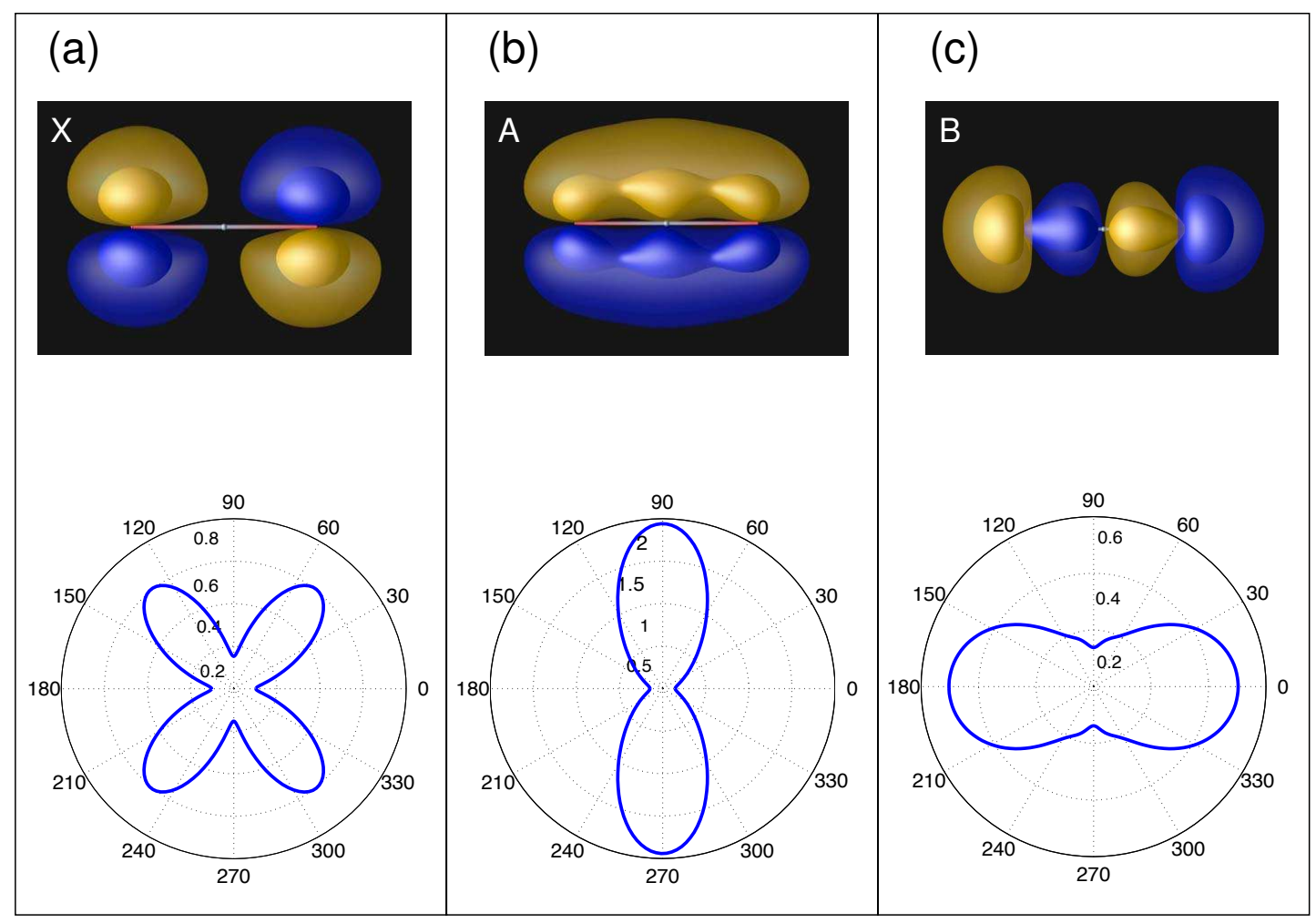

FIG. 2: Dyson orbitals and angular dependences of strong-field ionization rates for different final states of $\mathrm{CO}_{2}^{+}$, normalized to a single atomic orbital with the same ionization potential. Blue and yellow colors correspond to positive and negative lobes of the orbital. (a) Ionization with ion left in the ground state $\tilde{X}^{2} \Pi_{g}$, channel $X$. (b) Ion left in the first excited electronic state $\tilde{A}^{2} \Pi_{u}$, channel $A$. (c) Ion left in the second excited electronic state $\tilde{B}^{2} \Sigma_{u}^{+}$, channel $B$.

$I \sim 10^{14} \mathrm{~W} / \mathrm{cm}^{2}$. Distortions of the reduced single-electron density by the static external field at these intensities also remain small. The Stark shifts of the neutral and the ionic states are included in our calculations.

Response of the bound states to the oscillating laser field is fully adiabatic in the neutral $\mathrm{CO}_{2}$, since electronic excitations lie far in the UV range. However, non-adiabatic dynamics could be present in the ion due to relative proximity of the $\tilde{A}$ and $\tilde{B}$ states to the ground state. We use the multi-electron wavefunctions of the ionic states to calculate the transition dipole moments: $d_{X A}=0.46 \times \cos (\theta), d_{X B}=0.27 \times \sin (\theta)$. This allows us to describe laserinduced dynamics of the ion between ionization and recombination, solving time-dependent Schroedinger equation in the restricted basis of the $\tilde{X}, \tilde{A}$ and $\tilde{B}$ states and finding amplitudes 

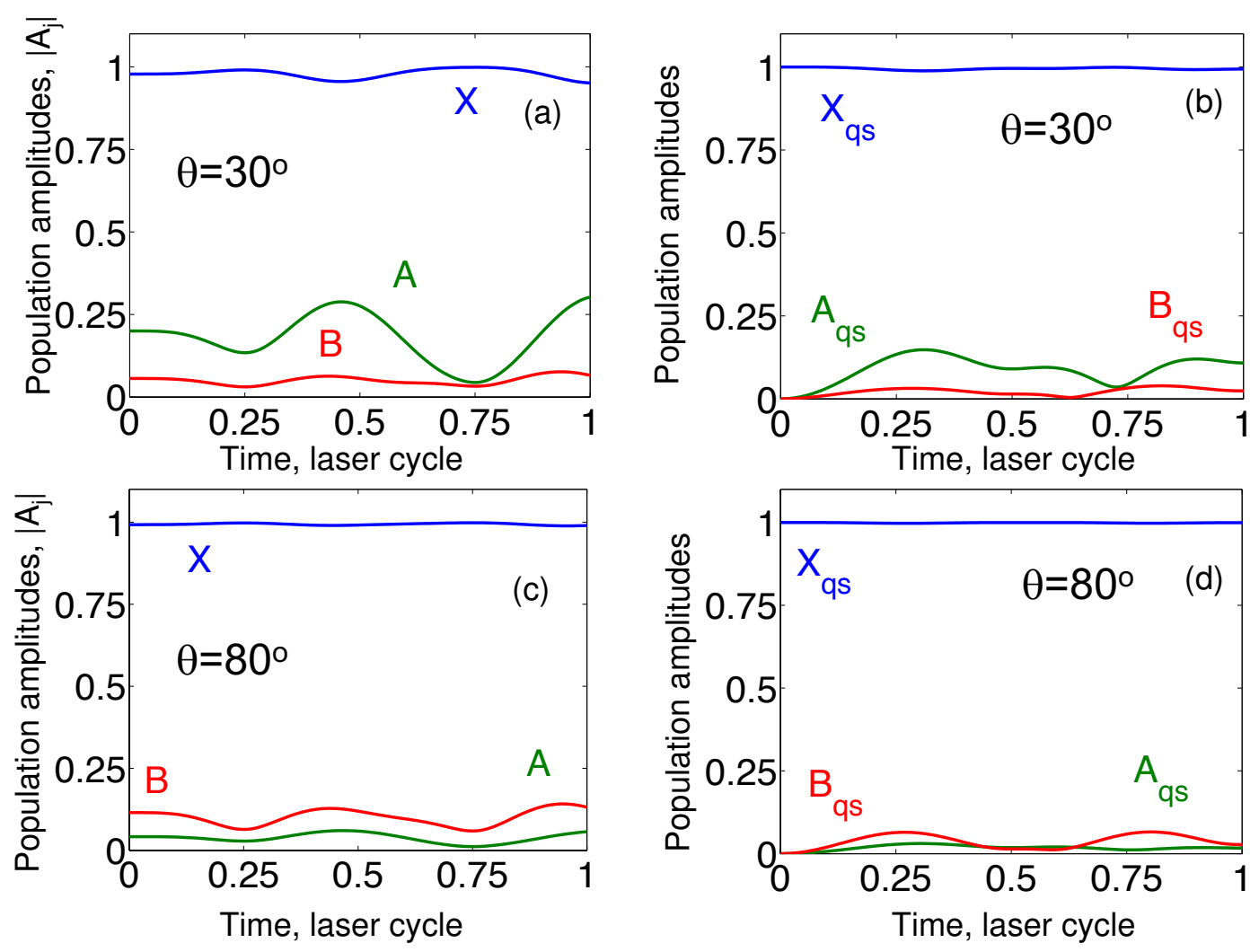

FIG. 3: The effect of the laser field $\left(1.72 \times 10^{14} \mathrm{~W} / \mathrm{cm}^{2}\right)$ on bound electrons in the ion. Subcycle amplitudes $\left|A_{j}(\theta, t)\right|$ (see Eq.1) of population of different ionic states between ionization and recombination for $\theta=30^{\circ}$ (a) and $\theta=80^{\circ}$ (c). The initial condition corresponds to leaving the ion in the lowest quasistatic state and the initial amplitudes in each channel are given by projections of the lowest quasistatic state onto field free states of the ion. (b,d) Amplitudes of populations of quasistatic states.

$A_{j}(\theta, t)$ (see Eq.1). Fig. 3 illustrates the effect of the laser field on bound electrons in the ion for one cycle of $800 \mathrm{~nm}$ laser field $F(t)=F_{0} \cos (\omega t), F 0=0.07$ a.u. $\left(1.72 \times 10^{14}\right.$ $\mathrm{W} / \mathrm{cm}^{2}$ ). Fig. 3(a,c) shows amplitudes $\left|A_{j}(\theta, t)\right|$ for small angles $\theta$ (Fig. 3(a), $\theta=30^{\circ}$ ) and large angles $\theta$ (Fig. 3(c), $\theta=80^{\circ}$ ) of molecular alignment. Note that for small angles the most important contribution to harmonic emission comes from channels $\mathrm{X}$ and $\mathrm{B}$, for large angles from channels $\mathrm{A}$ and $\mathrm{X}$. The initial condition corresponds to leaving the ion in the lowest quasistatic state and the initial amplitudes in each channel are given by projections of the lowest quasistatic state onto field free states of the ion. Quasistatic states are found by diagonalization of three level system $(\mathrm{X}, \mathrm{A}, \mathrm{B})$ in static field. The initial populations in states 
$\mathrm{A}$ and $\mathrm{B}$ reflect laser induced polarization in the ion. To analyze sub -cycle laser induced dynamics in the ion it is instructive to consider the population amplitudes (square roots of probabilities) of the quasistatic states (Fig 3(b) for $\theta=30^{\circ}$ and Fig $3(\mathrm{~d})$ for $\theta=80^{\circ}$ ). These show that the laser induced transitions between the quasistatic states are small for both channel B at small angles and channel A for large angles. Therefore, we conclude that the effect of the laser field on bound electrons is dominated by polarization rather than laser-induced non-adiabatic transitions between the quasistatic states.

\section{PROPAGATION AMPLITUDE}

Each moment of recombination $t$ corresponds to the moment of 'birth' (ionization) $t_{b}$ at which the electron appears in the continuum with (nearly) zero velocity component along the laser field. Following standard approach [3], the propagation amplitude is approximated as

$$
a_{\text {prop }}\left[t, t_{b}, \theta\right] \propto\left(\frac{1}{\left(t-t_{b}-i \tau\left(t_{b}\right)\right)^{1 / 2}}\right)\left(\frac{1}{v_{\perp}\left(t-t_{b}-i \tau\left(t_{b}\right)\right)}\right) F_{\perp}(\theta)
$$

Here $\tau\left(t_{b}\right)$ is the Keldysh-type sub-cycle tunnelling time obtained following the approach described in Ref. [7]. The first fraction describes longitudinal stretching of the wavepacket between the moments of ionization and recombination (see [3]). The second fraction describes wavepacket spreading perpendicular to the laser polarization, with spreading velocity $v_{\perp}$. For well-localized initial states it is given by [11] $1 / v_{\perp}=\sqrt{\tau}$, in agreement with the energy-time uncertainty relationship. To take into account characteristic transverse size $\Delta r_{\perp}$ of the ionizing state, we use the approximation $1 / v_{\perp} \propto \sqrt{\tau+\Delta r_{\perp}^{2}}$, which combines the energy-time and the coordinate-momentum uncertainty relationships. The contribution to the spreading velocity due to characteristic size of the Dyson orbital has been determined directly from the Fourier transform of the orbital. Correction to the connection between $t$ and $t_{b}(t)$ due to the attractive long-range Coulomb tail of the ionic potential is included as described in [4]. The angle-dependent factor $F_{\perp}(\theta)$ includes the contribution of the nodal planes of the ionizing orbital to the transverse shape of the continuum wavepacket. 


\section{SCATTERING STATES IN THE STRONG LASER FIELD.}

To find the required wavefunction of the scattering state with asymptotic momentum $k(t)$, we substitute the ansatz

$$
\Psi_{C, j}(t, \theta)=\hat{A} \Psi_{j}^{(t, \theta)} \chi_{C, j}(t, \theta)
$$

into the time-dependent $N$-electron Schroedinger equation. Following [13], we obtain equation for the continuum wavefunction

$$
i \dot{\chi}_{C, j}=\left[-\frac{\Delta}{2}+V_{L}(t)+U_{\text {Hartree,j }}(t)+U_{\text {nuclei }}(t)\right] \chi_{C, j}
$$

where $V_{L}$ is the interaction with the laser field, $U_{\text {Hartree,j }}(t)+U_{\text {nuclei }}$ is the multiplicative part of the electron interaction potential, consisting of the Coulomb potential of the nuclei (C-O distance equal to $1.1621 \AA$ ), and the Hartree potential of the core electrons for the ionic state corresponding to the channel $j$. The non-local exchange contributions are omitted in Eq.(5). Equation (5) is solved using strong-field eikonal-Volkov aproximation, SF-EVA [4]. Additionally, the adiabatic approximation, which ignores the Coulomb-laser coupling, is used as described in [12],[4]. The Hartree potential, which is required to calculate the SFEVA continuum wavefunction, was found by the numerical solution of the Poisson equations on a uniformly spaced $(\mathrm{d}=0.116 \stackrel{\circ}{A})$ numerical grid in a $69.7 \AA$ cubic box.

We find $k(t)$ by setting the (asymptotic) kinetic energy of the returning electron $E_{\text {kin }}(t)=$ $k(t)^{2} / 2$ to that acquired by the electron from the laser field. It includes important correction to the standard term $\left[A(t)-A\left(t_{b}\right)\right]^{2} / 2: \quad E_{\text {kin }}(t)=\left[A(t)-A\left(t_{b}\right)\right]^{2} / 2+\Delta E(t)$ and hence $k(t)=A\left(t_{b}\right)-A(t)+\Delta k(t)$. Here $A(t)$ is the vector-potential of the laser field, with electric field $E(t)=-\partial A(t) / \partial t$, and $t_{b}=t_{b}(t)$ is the moment of 'birth' (ionization) that corresponds to the moment of recombination $t$. Mathematically, the correction $\Delta E$ appears automatically when one accounts for the imaginary part of the complex 'quantum trajectory' (saddle point of the time-integrals) that connects $t$ and $t_{b}$. For technical convenience, we separate the motion along the 'quantum trajectory' into the motion along the real time axis, which corresponds to the classically allowed motion after tunnelling, and the motion along the imaginary time axis, which corresponds to tunnelling through the classically forbidden region. The time of birth $t_{b}$ is defined as the real part of the complex saddle point, and the additional recollision energy due to the imaginary part is written explicitly as $\Delta E$. 
Physically, this correction comes from the fact that after ionization the characteristic electron trajectory starts at a distance $z\left(t_{b}\right)$ away from the origin. For arbitrary phase of birth, this distance is given in [4]. The liberated electron has to cover this extra distance on the way back before recombination. Additional energy $\Delta E$ acquired by the electron from the laser field over this distance increases the electron recollision energy for short trajectories. Near the cutoff, this extra energy equals $0.32 I_{p}[14]$, and follows automatically from our calculations (in the exact same way as it has appeared in the original paper[14]). For ionization potentials of $I_{p} \sim 15-19 \mathrm{eV}$ the corresponding correction of $5-6 \mathrm{eV} \sim 3-4 \omega$ is significant.

We also stress that in our calculation there is no need to artificially adjust the so-called 'dispersion relationship' between the asymptotic electron kinetic energy $E_{\text {kin }}(t)=k(t)^{2} / 2$ and the energy of the emitted photon. Indeed, the SF-EVA approximation naturally accounts for the acceleration of the returning electron by the potential of the ionic core potential. This relationship is automatically enforced by the Fourier transform of the timedependent dipole, and agrees with the energy conservation law $N \omega=I_{p}+k\left(t_{N}\right)^{2} / 2$. Here $t_{N}$ is the moment of recombination corresponding to the emission of $N$-th harmonic.

Using the SF-EVA scattering states, we construct the $N$-electron wavefunction using the ansatz of Eq.(4) and calculate the recombination matrix elements with the $N$-electron wavefunction of the ground state. The ansatz Eq.(4) includes electron exchange corrections in the matrix elements [15]. Channel couplings during recombination are not included.

\section{CONTRIBUTION OF DIFFERENT COMPONENTS OF ELECTRON-ION WAVEPACKET TO DYNAMICAL MINIMA.}

Dynamical interference minima in harmonic amplitudes occur when channels contributing to the harmonic signal interfere destructively. The phase of each channel has three main contributions: the phase accumulated in the ion $E_{j} \tau_{j}$, the Volkov phase of the continuum electron, the phase of the recombination dipole matrix element and the phase acquired during ionization ( see Section 13). Here $\tau_{j}$ is the 'travel' time - the time-delay between ionization and recombination for the channel $\mathrm{j}$. For the same harmonic number, the phases accumulated in the ion differ by $\approx\left(E_{2}-E_{1}\right) \tau$, where $\tau$ is the average travel time for the two channels. The Volkov phases for channels $j=1,2$ differ much less, only by $\sim\left(E_{2}-E_{1}\right) \Delta \tau$. Here $\Delta \tau=\tau_{2}-\tau_{1}$ is the difference between travel times for the two channels. Compared to 
$\left(E_{2}-E_{1}\right) \tau$, relative phases of the recombination matrix elements for the two channels change slowly as a function of harmonic number, except for the vicinity of the structural minimum for a specific channel, where the corresponding recombination matrix element switches the sign.

\section{SELECTION OF 'SHORT' TRAJECTORIES.}

In single-molecule response, there are several so-called 'quantum' trajectories of the liberated electron that return to the parent ion with the same electron energy but at different times $t$, see e.g. Ref.[16]. These trajectories are grouped into 'short' and 'long', depending on whether they return to the parent ion before or after the highest-energy trajectory with the energy $\sim 3.2 U_{p}$ (here $U_{p}$ is the average oscillation energy of the free electron in the laser field)[16].

However, in a molecular gas propagation of harmonic radiation associated with short and long trajectories is different, in terms of both the phase-matching and the divergence. In typical experiments on high harmonic generation, the geometry of the experimental setup suppresses the so-called 'long' trajectories and favors the short ones. To account for this experimental aspect, we (i) first calculated $D(t)$ for each ionization burst (i.e. each halfcycle) and then (ii) multiplied it with a temporal Gaussian filter. The filter smoothly suppresses the signal after the most energetic $3 U_{p}$ trajectory, for each ionization burst.

\section{AVERAGING OVER ALIGNMENT DISTRIBUTION.}

In experiments, molecules are pre-aligned with an aligning pulse. When the aligning and the harmonic-generating laser pulses are polarized parallel to each other, the typical degree of alignment for the molecular ensemble is $\pm 30^{\circ}$ in the standard experimental conditions. This degree of alignment is well-reproduced by either $\cos ^{4} \theta$ or $\cos ^{6} \theta$ distributions (the former having characteristic alignment angle of $\sim 32^{\circ}$ and the latter $\sim 28^{\circ}$.) In our calculations, angle-averaging of the emitted light (i.e. Fourier transform of $D(t)$ ) was performed with $\cos ^{4} \theta, \cos ^{6} \theta$, and $\cos ^{10} \theta$ distribution, to examine the role of angle-averaging. All of our conclusions are robust with respect to the model distribution chosen for angular averaging. 


\section{INTENSITY CALIBRATION}

Experimental intensities were calibrated by comparing observed and calculated cutoff positions in the harmonic spectra. We have also additionally checked that the extracted intensity satisfies the harmonic cutoff rule Eq.(6). The cutoff is determined by the maximum recollision energy $E_{e}$ plus the ionization potential $I_{p}[14]$ :

$$
\Omega_{\text {cutoff }}=3.17 U_{p}+1.32 I_{p}
$$

Here $U_{p}=\mathcal{E}^{2} / 4 \omega^{2}$ is the average electron oscillation energy, $\mathcal{E}$ and $\omega$ are the laser field strength and frequency, and $3.17 U_{p}$ is the maximum energy the electron picks up between ionization and return to the place of its 'birth' in the continuum[17]. The additional energy $0.32 I_{p}$ is picked up by the returning electron in the strong-field regime, due to the offset of the point of tunnel ionization ('birth') from the origin[14]. Note that for the B channel in $\mathrm{CO}_{2}$, the ionization energy is $4.3 \mathrm{eV}$ higher than that for the $\mathrm{X}$ channel, leading to the corresponding shift of the harmonic cutoff by nearly $6 \mathrm{eV}$. As a result, the $\mathrm{B}$ channel in $\mathrm{CO}_{2}$ dominates the cutoff emission for molecules aligned at small angles $\theta$, while the A channel is significant for perpendicular alignment, $\theta \sim 90^{\circ}$.

Finally, we also checked that our value of the calibrated intensity agrees with the intensity calibration in the experiments[18], for the position of the minimum at H23.

We stress that our reconstruction of the hole dynamics relies firmly on the experimental measurements, while the theoretical calculations lend further support to our interpretation.

\section{ACCURACY OF PHASE RECONSTRUCTION}

First, we note that position of the minimum depends linearly on intensity if the relative phase between the channels $\phi$ is constant (i.e. intensity-independent).

Setting $\phi=-\pi / 2$ brings the moment of destructive interference $\tau^{*}$ earlier in time. Indeed, then the initial ionic wavepacket $\Psi_{X}+e^{-i \pi / 2} \Psi_{B}$ and its further evolution is $\Psi_{X}+e^{-i \pi / 2-i\left(E_{B}-E_{X}\right) \tau} \Psi_{B}$. The relative phase that needs to be accumulated during the wavepacket evolution in the ion, to yield the destructive interference between the channels, is therefore reduced by $\pi / 2$. The harmonic suppressed due to channel interference is therefore emitted at a shorter time-delay $\tau^{*}$. For 'short' trajectories, both the corresponding 
harmonic number is lower and the energy of the electron at this moment is smaller (yielding smaller slope of the intensity dependence).

Setting $\phi=+\pi / 2$ moves the moment of destructive interference $\tau^{*}$ later in time. Thus, both the corresponding harmonic number is higher and the energy of the electron at this moment is higher (bigger slope of the intensity dependence). However, we stress that if the initial phase of ionization changes with intensity, the intensity dependence becomes nonlinear.

Fig. 4 shows the positions of the calculated minima for $\phi=-\pi / 2, \phi=0$ and $\phi=+\pi / 2$, for all intensities. These figures demonstrate that at higher intensities the experimental data can reliably distinguish between $\phi=-\pi / 2, \phi=+\pi / 2$, and $\phi=0$.

\section{PHASE OF IONIZATION IN THE MULTIPHOTON REGIME.}

The question of the relative phase $\phi$ between different channels in strong-field ionization has not been addressed before. For single ionization channel, this question is meaningless, and the importance of multiple ionization channels in optical tunnelling has been underestimated so far. Even for several ionization channels the relative phase cannot be measured in photo-electron spectra of direct ionization: if the ion is left in different (orthogonal) states, the correlated electron wavepackets do not interfere.

We expect that the phase $\phi$ will depend on the ionization regime. Experimental data (see Fig.5(c) in the main body of the paper) seem to suggest that at lower intensities, when the ionization regime changes from tunnelling to multiphoton at $I \approx 1.1-1.2 \times 10^{14} \mathrm{~W} / \mathrm{cm}$, the intensity dependence of the minimum might be deviating from linear. One possible interpretation is the intensity dependence of $\phi$. At $I \approx 5 \times 10^{13} \mathrm{~W} / \mathrm{cm}^{2}$ the harmonic minimum is found at H19, lower than our tunnelling-based calculation predicts. Setting $\phi=$ $-\pi / 2$ shifts the theoretically calculated minimum to H17-H19. This phase yields maximal charge delocalization for the participating channels (Fig.5(e), snapshot (2)). Indeed, in the multi-photon regime the electric field cannot be considered static during ionization and the preference between the two opposite directions of ionization is lost. However, the deviation 

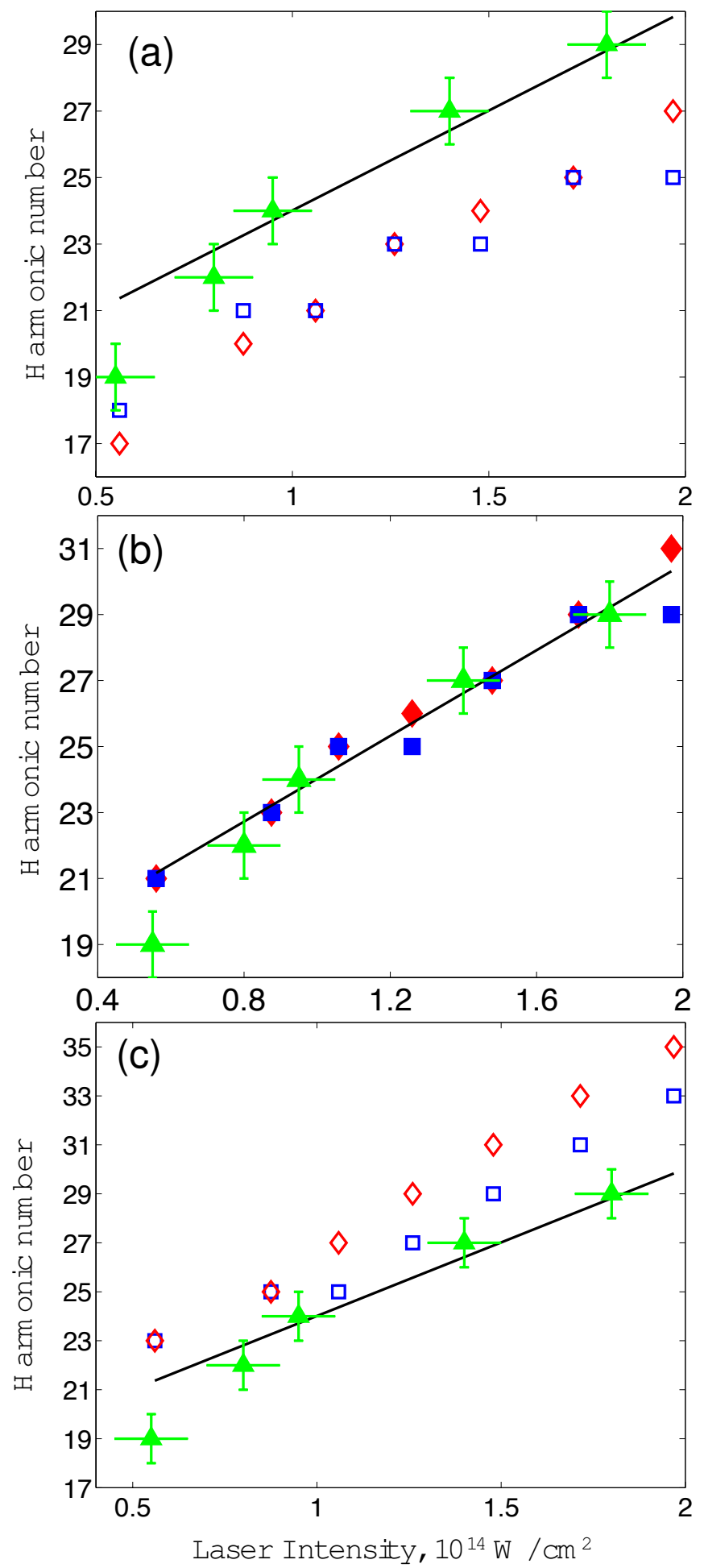

FIG. 4: Comparison of the experimental (triangles) and theoretical results for the position of the minimum as a function of intensity. Theoretical results are averaged over $\cos ^{4} \Theta$ (diamonds) and $\cos ^{6} \Theta$ (squares) angular distributions for aligned molecules. Experimental data are shown as triangles. Panel (a) shows results of calculations with $\phi=-\pi / 2$, panel (b) shows shows results of calculations with $\phi=0$, panel (c) shows results of calculations with $\phi=+\pi / 2$. 
from $\phi=0$ at the lowest intensity is at the limits of the accuracy of our reconstruction.

[1] Salières, P. et al., Science 292, 902 (2001).

[2] Bartels, R. A. et al., Phys. Rev. Lett. 88, 013903 (2001).

[3] M. Yu. Ivanov, T. Brabec, and N. H. Burnett, Phys. Rev. A 54, 742-745 (1996)

[4] O. Smirnova, M. Spanner and M. Yu. Ivanov, Phys. Rev. A, 77, 033407, (2008)

[5] Y Mairesse et al, New J. of Physics, v. 10, 025015 (2008)

[6] A. Becker and F. Faisal, J. Phys. B 38 R1 (2005)

[7] G. Yudin and M. Yu. Ivanov, Phys. Rev. A, 64, 013409 (2001)

[8] J. Muth-Böhm, A. Becker, F. H. M. Faisal, Phys. Rev. Lett. 85, 2280 (2000).

[9] A. Jaron-Becker, A. Becker, F.H.M. Faisal, J. Phys. B 36 L375 (2003)

[10] A. Jaron-Becker, A. Becker, F.H.M. Faisal, Phys. Rev. A, 69, 023410 (2004)

[11] M. Yu. Ivanov, O. Smirnova, and M. Spanner, Journal of Modern Optics, 52, 165 (2005)

[12] O. Smirnova et al., J. Phys. B, 40, F197 (2007)

[13] S. Tonzani, C. H. Greene J. Chem. Phys. 122, 014111 (2005)

[14] M. Lewenstein et al, Phys. Rev. A, 49, 2117 (1994).

[15] S. Patchkovskii et al., Phys. Rev. Lett. 97, 123003 (2006).

[16] P. Agostini, L. F. DiMauro Rep. Prog. Phys. 67, 813 (2004), Nature Physics, v. 4, p. 545, (2008)

[17] Corkum, P. B. Phys. Rev. Lett. 71, 1994 (1993).

[18] Boutu, W. et al. Nature Physics, v. 4, p. 545, (2008) 